\title{
Review Article \\ Serrated Polyps and Their Alternative Pathway to the Colorectal Cancer: A Systematic Review
}

\author{
Lukasz Szylberg, ${ }^{1}$ Marlena Janiczek, ${ }^{1}$ Aneta Popiel, ${ }^{1}$ and Andrzej Marszałek ${ }^{1,2}$ \\ ${ }^{1}$ Department of Clinical Pathomorphology, Collegium Medicum in Bydgoszcz, Nicolaus Copernicus University in Torun, \\ Sklodowskiej-Curie 9, 85-094 Bydgoszcz, Poland \\ ${ }^{2}$ Department of Oncologic Pathology, Poznan University of Medical Sciences, Poznan, Poland
}

Correspondence should be addressed to Andrzej Marszałek; amars@ump.edu.pl

Received 25 November 2014; Revised 20 March 2015; Accepted 22 March 2015

Academic Editor: Cristiano Pagnini

Copyright (C) 2015 Łukasz Szylberg et al. This is an open access article distributed under the Creative Commons Attribution License, which permits unrestricted use, distribution, and reproduction in any medium, provided the original work is properly cited.

\begin{abstract}
Colorectal cancer (CRC) is the third most frequently diagnosed cancer in the world. For a long time, only one pathway of colorectal carcinogenesis was known. In recent years, a new "alternative" pathway through serrated adenoma was described. Recent metaanalysis estimated these cancers as about $10 \%$ to $30 \%$ of all CRCs. Serrated polyps are the second most popular groups of polyps (after conventional adenomas) found during colonoscopy. Serrated polyps of the colon are clinically and molecularly diverse changes that have common feature as crypt luminal morphology characterized by glandular serration. Evidence suggests that subtypes of serrated polyps, particularly TSA and SSA/P, can lead to adenocarcinoma through the serrated pathway. Moreover, the data indicate that the SSA/P are the precursors of colorectal carcinoma by MSI and may be subject to rapid progression to malignancy. An important step to reduce the incidence of CRC initiated by the serrated pathway is to improve the detection of serrated polyps and to ensure their complete removal during endoscopy. Understanding of the so-called serrated carcinogenesis pathway is an important step forward in expanding possibilities in the prevention of CRC.
\end{abstract}

\section{Introduction}

Colorectal cancer (CRC) is the third most frequently diagnosed cancer in the world. Each year about 600,000 people die from it [1]. Over $90 \%$ of CRCs are adenocarcinomas [2]. For a long time, only one pathway of colorectal carcinogenesis was known. Vogelstein et al. described it as "classical" pathway through adenoma-adenocarcinoma sequence [3]. In recent years, a new "alternative" pathway through serrated adenoma was described. Adenocarcinomas developed from serrated lesions were first described by Jass and Smith [4]. They estimated these cancers as about $10 \%$ to $30 \%$ of all CRCs. Serrated polyps are the second most popular groups of polyps (after conventional adenomas) found during colonoscopy [5]. In the literature two terms are used and accepted for serrated lesions such as serrated polyps or serrated adenomas [6]. According to the 2010 WHO classification, serrated polyps are divided into three subgroups such as sessile serrated adenoma/polyp (SSA/P), traditional serrated adenoma (TSA), and hyperplastic polyps (HPs) [7]. HP has been recognized as a distinct type of these lesions in the colon. In recent years, it has been challenged that HP was considered as benign lesion, but numerous studies have shown the malignant nature of this lesion. In this regard, nature of HP is clinically important. The appearance of this type of polyp in the colon should lead to oncological alert and obligatory procedure of polyp removal [8]. Serrated pathway leading to the development of colon cancer is not homogeneous. It depends on the genetic and molecular variation [9]. Understanding carcinogenesis pathways can improve the treatment process and help prevent the CRC development.

This review presents the current knowledge of serrated polyps and their pathways leading to CRC. Serrated polyps are interdisciplinary problem among gastroenterologists, pathologists, and oncologists.

\section{Classification of Serrated Polyps}

2.1. General Classification. Serrated adenomas were first described in 1990 by Longacre and Fenoglio-Preiser [10]. Serrated polyps are heterogeneous lesions. Histologically they 
are characterized by glandular serration. Colonic epithelial crypts show luminal "saw-toothed" pattern. This feature is considered as a result of cell growth in combination with the rotation of the delayed migration or failure of cells apoptosis which lead to accumulation of the epithelial cells. It is now recognized that several different subtypes of serrated polyps exist and can lead to a subset of invasive cancer by serrated pathway [11]. Serrated polyp nomenclature is in evolution. The latest classification of the World Health Organization (WHO) classifies them into three main groups: HP, SSA/P, and TSA [12].

2.1.1. Hyperplastic Polyps. HP represents more than threequarters of serrated polyps [13]. HPs are flat or sessile, pale lesions, generally do not exceed $5 \mathrm{~mm}$, and are usually located at the ends of the folds of the rectal mucosa. In colorectum, HPs are often larger and more difficult to visualize in endoscopic procedure. HPs develop at a younger age than conventional adenomas, but their frequency does not seem to significantly increase after 50 years of age [14].

2.1.2. Sessile Serrated Adenomas/Polyps. SSA were first identified in 2003 by Torlakovic et al. [15]. They Represent 15 to $20 \%$ of all serrated polyps [16]. SSA are flat or slightly elevated. These lesions occur most commonly in the proximal colon and are typically measuring more than $5 \mathrm{~mm}$. Histologically, SSA differ from HP, the presence of abnormal architecture characteristics secondary to abnormal proliferation. In HP, the proliferation zone is located at the bottom of the crypts. However, in SSA, crypts proliferation leads to an increase in the crypts being asymmetric of T-shaped or inverted Lshaped structures. Other characteristic features include the presence of mature goblet cells at the base of the crypts, hyperserration throughout the base or in the crypts, and muscle pseudoinvasion. Dysplasia is absent $[6,17,18]$.

2.1.3. Traditional Serrated Adenomas. TSAs are relatively not very frequent polyps estimated up to $5 \%$ serrated polyps in Western countries with higher prevalence in Asia, especially in Korea [19]. Compared to the SSA, TSAs are found on the left side of the colon and in the elderly. The architecture of TSA is often more complicated than villous or tubulovillous adenoma, but with visible serration. Ectopic crypt foci are present in the TSA, specified by presence of crypts at their base (not sitting at the level of muscularis propria). This morphological feature is useful to distinguish them from the SSA. Neoplastic cells are characterized by abundant eosinophilic cytoplasm and elongated, pencillate nuclei. Dysplasia of TSA is usually clinically "benign" comparing appearance of dysplasia associated with conventional adenoma and low proliferation properties [20].

\section{Epigenetic and Genetic Aspects of Serrated Polyps}

Classical "adenoma-carcinoma" sequence which includes changes from the normal mucosa towards the carcinoma belongs to specific and well defined genetic alterations such as
APC (adenomatous polyposis coli), and oncogenes as KRAS (Kristen rat sarcoma viral oncogene homolog), DCC (deleted in CRC), and TP53 (tumor protein 53) [21]. Development of colon cancer is caused by a cascade of genetic mutations which leads to a progressive disordered DNA replication and accelerated colonocyte replication. Progressive cascade of genetic mutations causes the transformation from normal mucosa through benign adenomas to adenomas with high grade dysplasia and finally into invasive adenocarcinoma $[18,22]$. These four stages of CRC carcinogenesis are characterized by excessive activation of oncogenes and inactivation of tumor suppressor genes. In the first phase, inactivation of the APC gene causes the development of adenomas. In the second phase, KRAS mutations promote adenoma growth. In the third phase, the $\mathrm{LOH}$ (loss of heterozygosity) supports the progression of adenoma. In the last step, inactivation of TP53 triggers the final transition to the cancer. This sequence is present in $60 \%$ of CRC cases [18]. It was considered that about $20 \%$ of CRCs develop on the base of serrated lesions through another pathway called "serrated pathway." It is associated with the sequence of genetic and epigenetic alternations [23]. According to Torlakovic et al. [15], there are two main types of genetic sequences with their subtypes which lead to SAC (serrated adenocarcinoma) (Figure 1). Before 2003, serrated adenoma which includes TSA and SSA was named as hyperplastic polyps. SSA revealed BRAF mutation, high level of CpG island methylation (CIMP-high), and MLH1 gene hypermethylation $[24,25]$. Non-CIMP was attributed to two groups. The first one is closely associated with presence of TP53 mutations and location of the cancer in distal colon. The second one is characterized by low periodicity of hypermethylation and gene mutation which is specified for the cancer located usually in rectum [26]. Methylation of CpG islands is a proper way of reducing gene expression (more methylation means less expression). If the silenced gene is tumor suppressor gene, then loss of function simplifies carcinogenesis. Epigenetics describes the study of dynamic alterations in the transcriptional potential of a cell but does not engage in DNA sequence. Contemporary role of epigenetic events leads to development of diagnostic tests. It can be used in prediction of biological aggressiveness, proper diagnosis, and clinical response of certain cancers [27]. Identification of DNA methylated markers by methylation-specific PCR (MSP) or non-MSP prescribed how PCR primer should be designed. Epigenetic alterations often rather than genetic changes could be used as early diagnostic tools. There are useful early methylated genes such as SLC5A8, MINT1, MINT31, SFRP1, SFRP2, CDH13, CRBP1, RUNX3, p14ARF, HLTF, ITGA4, and CDKN2A. The hypermethylation promoter can be detected from tissue biopsies, blood samples, stool, peritoneal fluid, and urine [28]. Moreover, epigenetic alterations may influence drug resistance. An interesting example is 5fluorouracil (FU), which is conventional chemotherapeutic agent used for CRC treatment. 5-FU works by initiation apoptosis through several apoptosis regulatory genes. If targeted genes are silenced by DNA methylation in CRC cells, the lower response to 5-FU therapy was observed [29]. Silencing gene by methylation is an epigenetic event [30]. CIMP-high is clinically associated with older age, female sex, 


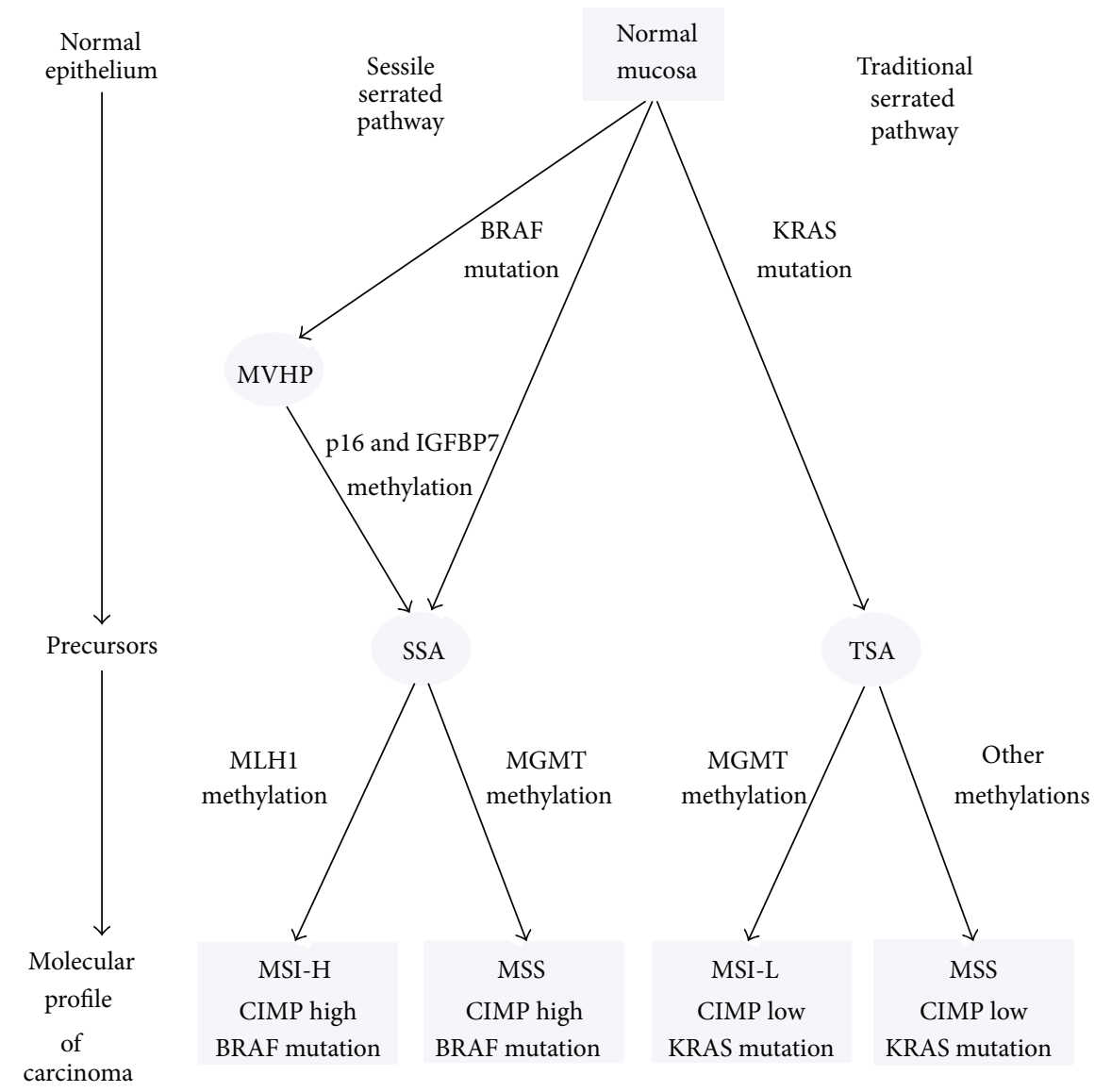

FIGURE 1: Sessile serrated and traditional serrated pathways. The first pathway consists of molecular profile cancers which are CpG island methylator phenotype-high (CIMP-high) and BRAF mutation positive with microsatellite instability being high (MSI-H) or stability (MMS). The precursor of these cancers may be MVHP and SSA. The second pathway consists of CpG island methylator phenotype-low (CIMP-low), KRAS mutations positive with microsatellite stability or instability being low (MSI-L). The precursor lesion in this second pathway may be TSA. MVHP: microvesicular hyperplastic polyp; SSA: sessile serrated adenoma; TSA: traditional serrated adenoma.

and proximal tumor location [31]. According to presence of MLH1-DNA mismatch repair gene is associated with MSI-H (microsatellite instability-high) and good prognosis. On the other hand, different type of methylation can lead to MSS (microsatellite stability) and has very poor prognosis [32]. TSAs are usually related to low grade dysplasia [14]. TSA is frequently associated with KRAS mutation but BRAF mutation can also occur. They are early molecular alterations in serrated lesions [33]. Additional feature of serrated pathway is silencing of the DNA repair gene O-6-methylguanine-DNA methyltransferase (MGMT) which is associated with CIMPlow. MGMT promoter methylation and silencing have been associated with guanine more than adenine mutations and MSI-low [34]. Based on Stefanius et al., BRAF mutation is more frequent than KRAS in all serrated adenocarcinomas. BRAF mutation is more specific to serrated adenocarcinomas than KRAS. KRAS mutation is linked with CIMP-low but is independent of MSI status [26]. BRAF or KRAS mutations may result in mitogen activated protein kinase (MAPK) activation. BRAF and KRAS mutation are among to the intracellular MAPK cascades responsible for cell growth [35]. However, many studies claimed that BRAF mutation is characteristic to the serrated pathway $[25,36]$. Colon cancers, which occurred after preventive colonoscopy, may represent cases such as missed cancers and development of cancers from missed or incompletely removed adenomatous polyps or rapid malignant progression of serrated polyps. Missed cancers have shown MSI and CIMP which they are both signature of serrated pathway. Serrated pathway of carcinogenesis could be related to duration of interval [37].

\section{Molecular Pathological Epidemiology of Serrated Lesions}

Molecular pathological epidemiology (MPE) is an integrative molecular and population health science, which is related to the molecular pathogenesis and variety of disease processes [38]. MPE provided contrast data to conventional epidemiological research which include genome-wide association studies (GWAS) [39]. The term "molecular pathological epidemiology" was used by Ogino and Stampfer in 2010 [40]. This term has been widely accepted by the scientists and clinicians $[41,42]$. MPE colorectal precancerous changes can provide unique opportunity to study effects of diet, lifestyle, and 
environmental factors on individual pathways of carcinogenesis. MPE lesions and intestines can identify causative exposures associated with the initiation and progression of cancer. It may help us to better understand CRC development and facilitate personalized prevention, screening, and treatment [38].

Recent research revealed the relation between the molecular pathology of CRC and exposure to risk factors. Samowitz and Limusi et al. demonstrated the relationship between CIMP-high and BRAF-mutated CRC and cigarette smoking $[43,44]$. Nishihara et al. showed that lower risk of CIMPhigh cancer is connected with former smokers with long term cessation [45]. Another study conducted by the BurnettHartman and colleagues focused on the relationship between the molecular pathology of serrated lesions and exposure to smoking cigarette. It has demonstrated statistical significance between CIMP-high and BRAF mutation and serrated lesions. They also observed the relationship between CIMP-low/negative and BRAF-wild-type serrated lesions and cigarette smoking. Therefore, the authors suggest that smoking may affect the initiation of serrated lesions, but not depending on BRAF mutation and CIMP. Another factor demonstrating the relationship between CIMP-high and BRAF mutations serrated lesions was high BMI [46]. However, in this case, no significant correlation with CRC was found $[47,48]$. Ethnicity and genetic variation are also evaluated in relation to molecular subsets of serrated lesions. The study showed that there is a positive association between CIMP-high in serrated lesions and Caucasians. Such correlation could potentially explain epidemiologic differences occurring between ethnic and racial groups [46]. However, in the study of colon cancer a clear relation to this factor has not been established $[49,50]$.

\section{Serrated Polyposis Syndrome}

Serrated polyposis syndrome (SPS) is now also known as hyperplastic polyposis syndrome described in the early seventies. This condition predisposes to cancer of the colon with the risk of $25-40 \%$ [51]. Although the first patients with SPS were documented in 1970, it has been recognized as the condition with genetic potential and precursor of CRC recently [52]. SPS are more common in people of Northern Europe and have relationships with cigarette smoking [53]. In addition to many serrated polyps, adenomas of the colon may be a part of this syndrome as they are identified in $85 \%$ of SPS patients [54]. SPS remains the least known and least understood changes in the colon. For a long time this syndrome was considered as having no clinical consequence, based on the opinion that serrated polyps are benign [55]. For decades it was thought that the malignant transformation of conventional adenomas is only a single mechanism underlying the development of CRC [56]. In the late nineties of the XX century, a number of important observations moving major paradigm shift in the way of initiation and progression of CRC [57]. These observations suggest that some serrated polyps may also act as precursor lesions in CRC development through alternative carcinogenesis pathways existing alongside traditional adenoma sequence. Observations suggest that a subset of serrated polyps can develop functions associated with cancer. The first clinical criteria for the recognition of serrated polyposis were created in 2000 [58]. This condition is determined to extract it from the familial adenomatous polyposis (FAP) and it became necessary to introduce appropriate criteria for observation of serrated polyps that are often in the distal part of the colon and rectum which did not include the definition of FAP [59]. The lesions in serrated polyposis do not exhibit the characteristics that distinguish them from sporadic serrated polyps, except that they are extremely numerous. The large polyps are often in the colon; small polyps are often seated distributed throughout the colon and rectum. The current revised criteria published in 2010 define serrated polyposis which includes any one of the following criteria: (a) at least five serrated polyps in the proximal sigmoid colon and at least two greater than $10 \mathrm{~mm}$, (b) any number of serrated polyps proximal to the sigmoid colon in first degree relative of patients with serrated polyposis, or (c) more than 20 serrated polyps of any size, but distributed throughout the colon [55]. However, the criteria for serrated polyposis differ in publications as some authors indicate large phenotypic changes and coincidence with sporadic polyps. Serrated polyposis is a relatively common disease and in such patients history of family CRC is relatively frequent phenomenon occurring up to $60 \%$ [60].

The serrated polyposis progression may be associated with premature aging of normal mucosa (e.g., the widespread gene promoter hypermethylation). Despite the large number of serrated polyps in the large intestine, only one-third has BRAF V600E mutation, a molecular feature of a serrated pathway. It suggests that the serrated lesions could develop from nonhomogeneous pathway $[36,61]$.

\section{Clinical Challenge Associated with Serrated Pathway}

Detailed knowledge of serrated pathway leading to CRC has important clinical implications for the detection, monitoring, and treatment [62]. Colorectal serrated polyps differ in clinical and molecular aspects which is reflected in different carcinogenesis pathway [63]. Unfortunately, the clinical data is limited, as it has been only a few years serrated that polyps are treated as potentially malignant lesions [64]. There are still many unanswered questions about the transition of serrated polyps into invasive cancer which differs from the classical adenoma-carcinoma sequence and whether its transformation is progressing faster or slower. Recent limited studies indicate that developing CRCs take place faster [65] and therefore it is important to remove these lesions during colonoscopy to prevent the development of invasive cancer. Cancer formed on the base of serrated lesion is mostly located in the proximal part of the large intestine. Serrated lesions have a distinct endoscopic appearance, but more difficult to diagnose than conventional polyps. Endoscopist should be trained in the diagnosis of serrated polyps to increase the level and effectiveness of colonoscopy. According to the recommendation of Rex et al. all serrated lesions proximal to the sigmoid colon and all serrated lesions in the rectosigmoid more 
than $5 \mathrm{~mm}$ in size should be completely removed. In addition, recommendations for postpolypectomy surveillance include monitoring patients with serrated lesions in colon and their families [17].

\section{Conclusion}

Understanding of the so-called serrated carcinogenesis pathway is an important step forward in expanding possibilities in the prevention of CRC. Serrated polyps of the colon are clinically and molecularly diverse changes that have common feature as crypt luminal morphology characterized by glandular serration. Evidence suggests that subtypes of serrated polyps, particularly TSA and SSA/P, can lead to adenocarcinoma through the serrated pathway. Moreover, the data indicate that the SSA/P are the precursors of colorectal carcinoma by MSI and may be subject to rapid progression to malignancy. SSA/P and MSI-H colorectal carcinomas are more common in the proximal colon. An important step to reduce the incidence of CRC initiated by the serrated pathway is to improve the detection of serrated polyps and to ensure their complete removal during endoscopy.

\section{Conflict of Interests}

The authors declare no conflict of interests regarding the publication of this paper.

\section{References}

[1] J. Ferlay, H.-R. Shin, F. Bray, D. Forman, C. Mathers, and D. M. Parkin, "Estimates of worldwide burden of cancer in 2008: GLOBOCAN 2008," International Journal of Cancer, vol. 127, no. 12, pp. 2893-2917, 2010.

[2] F. T. Bosman, R. H. Hruban, and N. D. Theise, WHO Classification of Tumors of the Digestive System, International Agency for Research on Cancer, Lyon, France, 2010.

[3] B. Vogelstein, E. R. Fearon, S. R. Hamilton et al., "Genetic alterations during colorectal-tumor development," The New England Journal of Medicine, vol. 319, no. 9, pp. 525-532, 1988.

[4] J. R. Jass and M. Smith, "Sialic acid and epithelial differentiation in colorectal polyps and cancer-a morphological, mucin and lectin histochemical study," Pathology, vol. 24, no. 4, pp. 233242, 1992.

[5] S. Yang, F. A. Farraye, C. Mack, O. Posnik, and M. J. O’Brien, "BRAF and KRAS mutations in hyperplastic polyps and serrated adenomas of the colorectum: relationship to histology and CpG island methylation status," American Journal of Surgical Pathology, vol. 28, no. 11, pp. 1452-1459, 2004.

[6] E. E. Torlakovic, J. D. Gomez, D. K. Driman et al., "Sessile serrated adenoma (SSA) vs. traditional serrated adenoma (TSA)," The American Journal of Surgical Pathology, vol. 32, no. 1, pp. 21-29, 2008.

[7] F. T. Bosman, World Helath Organisation, and International Agency for Research on Cancer, WHO Classification of Tumours of the Digestive System, International Agency for Research on Cancer, Lyon, France, 4th edition, 2010.

[8] V. P. Bauer and H. T. Papaconstantinou, "Management of serrated adenomas and hyperplastic polyps," Clinics in Colon and Rectal Surgery, vol. 21, no. 4, pp. 273-279, 2008.
[9] L. Yamane, C. Scapulatempo-Neto, R. M. Reis, and D. P. Guimarães, "Serrated pathway in colorectal carcinogenesis," World Journal of Gastroenterology, vol. 20, no. 10, pp. 26342640, 2014.

[10] T. A. Longacre and C. M. Fenoglio-Preiser, "Mixed hyperplastic adenomatous polyps/serrated adenomas. A distinct form of colorectal neoplasia," The American Journal of Surgical Pathology, vol. 14, no. 6, pp. 524-537, 1990.

[11] A. Ensari, F. T. Bosman, and G. J. A. Offerhaus, “The serrated polyp: getting it right!," Journal of Clinical Pathology, vol. 63, no. 8, pp. 665-668, 2010.

[12] D. Snover, D. J. Ahnen, R. W. Burt et al., "Serrated polyps of the colon and rectum and serrated ('hyperplastic') polyposis," in WHO Classification of Tumours. Pathology and Genetics. Tumours of the Digestive System, F. T. Bozman, F. Carneiro, R. H. Hruban, and etal, Eds., Springer, Berlin, Germany, 2010.

[13] R. J. Groff, R. Nash, and D. J. Ahnen, "Significance of serrated polyps of the colon," Current Gastroenterology Reports, vol. 10, no. 5, pp. 490-498, 2008.

[14] D. E. Aust and G. B. Baretton, "Serrated polyps of the colon and rectum (hyperplastic polyps, sessile serrated adenomas, traditional serrated adenomas, and mixed polyps)-proposal for diagnostic criteria," Virchows Archiv, vol. 457, no. 3, pp. 291-297, 2010.

[15] E. Torlakovic, E. Skovlund, D. C. Snover, G. Torlakovic, and J. M. Nesland, "Morphologic reappraisal of serrated colorectal polyps," The American Journal of Surgical Pathology, vol. 27, no. 1, pp. 65-81, 2003.

[16] N. S. Goldstein, P. Bhanot, E. Odish, and S. Hunter, "Hyperplastic-like colon polyps that preceded microsatellite-unstable adenocarcinomas," The American Journal of Clinical Pathology, vol. 119, no. 6, pp. 778-796, 2003.

[17] D. K. Rex, D. J. Ahnen, J. A. Baron et al., "Serrated lesions of the colorectum: review and recommendations from an expert panel," The American Journal of Gastroenterology, vol. 107, no. 9, pp. 1315-1329, 2012.

[18] F. Zoratto, L. Rossi, M. Verrico et al., "Focus on genetic and epigenetic events of colorectal cancer pathogenesis: implications for molecular diagnosis," Tumor Biology, vol. 35, no. 7, pp. 61956206, 2014.

[19] K.-M. Kim, E. J. Lee, Y.-H. Kim, D. K. Chang, and R. D. Odze, "KRAS mutations in traditional serrated adenomas from Korea herald an aggressive phenotype," The American Journal of Surgical Pathology, vol. 34, no. 5, pp. 667-675, 2010.

[20] R. K. Yantiss, K. Y. Oh, Y.-T. Chen, M. Redston, and R. D. Odze, "Filiform' serrated adenomas: a clinicopathologic and immunophenotypic study of 18 cases," The American Journal of Surgical Pathology, vol. 31, no. 8, pp. 1238-1245, 2007.

[21] K. Stefanius, Colorectal Carcinogenesis via Serrated Route, Acta Universitatis Ouluensis, Oulun Yliopisto, 2011.

[22] D. Colussi, G. Brandi, F. Bazzoli, and L. Ricciardiello, "Molecular pathways involved in colorectal cancer: implications for disease behavior and prevention," International Journal of Molecular Sciences, vol. 14, no. 8, pp. 16365-16385, 2013.

[23] E. Kaemmerer, C. Klaus, M. K. Jeon, and N. Gassler, "Molecular classification of colorectal carcinomas: the genotype-tophenotype relation," World Journal of Gastroenterology, vol. 19, no. 45, pp. 8163-8167, 2013.

[24] T. B. Sheridan, H. Fenton, M. R. Lewin et al., "Sessile serrated adenomas with low- and high-grade dysplasia and early carcinomas: an immunohistochemical study of serrated lesions 
'caught in the act'” American Journal of Clinical Pathology, vol. 126, no. 4, pp. 564-571, 2006.

[25] J. E. East, B. P. Saunders, and J. R. Jass, "Sporadic and syndromic hyperplastic polyps and serrated adenomas of the colon: classification, molecular genetics, natural history, and clinical management," Gastroenterology Clinics of North America, vol. 37, no. 1, pp. 25-46, 2008.

[26] T. Hinoue, D. J. Weisenberger, C. P. E. Lange et al., "Genomescale analysis of aberrant DNA methylation in colorectal cancer," Genome Research, vol. 22, no. 2, pp. 271-282, 2012.

[27] W. B. Coleman and A. G. Rivenbark, "Quantitative DNA methylation analysis the promise of high-throughput epigenomic diagnostic testing in human neoplastic disease," Journal of Molecular Diagnostics, vol. 8, no. 2, 2006.

[28] J. M.-K. Ng and J. Yu, "Promoter hypermethylation of tumour suppressor genes as potential biomarkers in colorectal cancer," International Journal of Molecular Sciences, vol. 16, no. 2, pp. 2472-2496, 2015.

[29] K. Bardhan and K. Liu, "Epigenetics and colorectal cancer pathogenesis," Cancers, vol. 5, no. 2, pp. 676-713, 2013.

[30] M. Toyota, N. Ahuja, M. Ohe-Toyota, J. G. Herman, S. B. Baylin, and J.-P. J. Issa, "CpG island methylator phenotype in colorectal cancer," Proceedings of the National Academy of Sciences of the United States of America, vol. 96, no. 15, pp. 8681-8686, 1999.

[31] W. S. Samowitz, H. Albertsen, J. Herrick et al., "Evaluation of a large, population-based sample supports a CpG island methylator phenotype in colon cancer," Gastroenterology, vol. 129, no. 3, pp. 837-845, 2005.

[32] Á. V. Patai, B. Molnár, Z. Tulassay, and F. Sipos, "Serrated pathway: alternative route to colorectal cancer," World Journal of Gastroenterology, vol. 19, no. 5, pp. 607-615, 2013.

[33] R. Beach, A. O.-O. Chan, T.-T. Wu et al., "BRAF mutations in aberrant crypt foci and hyperplastic polyposis," The American Journal of Pathology, vol. 166, no. 4, pp. 1069-1075, 2005.

[34] S. Ogino, T. Kawasaki, G. J. Kirkner, Y. Suemoto, J. A. Meyerhardt, and C. S. Fuchs, "Molecular correlates with MGMT promoter methylation and silencing support $\mathrm{CpG}$ island methylator phenotype-low (CIMP-low) in colorectal cancer," Gut, vol. 56, no. 11, pp. 1564-1571, 2007.

[35] K. Stefanius, L. Ylitalo, A. Tuomisto et al., "Frequent mutations of KRAS in addition to BRAF in colorectal serrated adenocarcinoma," Histopathology, vol. 58, no. 5, pp. 679-692, 2011.

[36] M. J. O'Brien, S. Yang, C. Mack et al., "Comparison of microsatellite instability, CpG island methylation phenotype, BRAF and KRAS status in serrated polyps and traditional adenomas indicates separate pathways to distinct colorectal carcinoma end points," The American Journal of Surgical Pathology, vol. 30, no. 12, pp. 1491-1501, 2006.

[37] S. Sweetser, T. C. Smyrk, and F. A. Sinicrope, "Serrated colon polyps as precursors to colorectal cancer," Clinical Gastroenterology \& Hepatology, vol. 11, no. 7, pp. 760-767, 2013.

[38] P. Lochhead, A. T. Chan, E. Giovannucci et al., "Progress and opportunities in molecular pathological epidemiology of colorectal premalignant lesions," The American Journal of Gastroenterology, vol. 109, no. 8, pp. 1205-1214, 2014.

[39] S. Ogino, P. Lochhead, A. T. Chan et al., "Molecular pathological epidemiology of epigenetics: emerging integrative science to analyze environment, host, and disease," Modern Pathology, vol. 26, no. 4, pp. 465-484, 2013.

[40] S. Ogino and M. Stampfer, "Lifestyle factors and microsatellite instability in colorectal cancer: the evolving field of molecular pathological epidemiology," Journal of the National Cancer Institute, vol. 102, no. 6, pp. 365-367, 2010.

[41] C. S. Ku, D. N. Cooper, M. Wu et al., "Gene discovery in familial cancer syndromes by exome sequencing: prospects for the elucidation of familial colorectal cancer type X," Modern Pathology, vol. 25, no. 8, pp. 1055-1068, 2012.

[42] M. Epplein, R. M. Bostick, L. Mu, S. Ogino, D. Braithwaite, and P. A. Kanetsky, "Challenges and opportunities in international molecular cancer prevention research: an ASPO Molecular Epidemiology and the Environment and International Cancer Prevention Interest Groups Report," Cancer Epidemiology Biomarkers \& Prevention, vol. 23, no. 11, pp. 2613-2617, 2014.

[43] W. S. Samowitz, H. Albertsen, C. Sweeney et al., "Association of smoking, CpG island methylator phenotype, and V600E BRAF mutations in colon cancer," Journal of the National Cancer Institute, vol. 98, no. 23, pp. 1731-1738, 2006.

[44] D. Limsui, R. A. Vierkant, L. S. Tillmans et al., "Cigarette smoking and colorectal cancer risk by molecularly defined subtypes," Journal of the National Cancer Institute, vol. 102, no. 14, pp. 1012$1022,2010$.

[45] R. Nishihara, T. Morikawa, A. Kuchiba et al., "A prospective study of duration of smoking cessation and colorectal cancer risk by epigenetics-related tumor classification," American Journal of Epidemiology, vol. 178, no. 1, pp. 84-100, 2013.

[46] A. N. Burnett-Hartman, P. A. Newcomb, J. D. Potter et al., "Genomic aberrations occurring in subsets of serrated colorectal lesions but not conventional adenomas," Cancer Research, vol. 73, no. 9, pp. 2863-2872, 2013.

[47] L. A. E. Hughes, C. C. J. M. Simons, P. A. van den Brandt et al., "Body size, physical activity and risk of colorectal cancer with or without the CpG island methylator phenotype (CIMP)," PLoS ONE, vol. 6, no. 4, Article ID e18571, 2011.

[48] M. L. Slattery, K. Curtin, C. Sweeney et al., "Diet and lifestyle factor associations with $\mathrm{CpG}$ island methylator phenotype and BRAF mutations in colon cancer," International Journal of Cancer, vol. 120, no. 3, pp. 656-663, 2007.

[49] M. L. Slattery, A. Lundgreen, J. S. Herrick, B. J. Caan, J. D. Potter, and R. K. Wolff, "Associations between genetic variation in RUNX1, RUNX2, RUNX3, MAPK1 and eIF4E and risk of colon and rectal cancer: additional support for a TGF- $\beta$-signaling pathway," Carcinogenesis, vol. 32, no. 3, pp. 318-326, 2011.

[50] D. R. English, J. P. Young, J. A. Simpson et al., "Ethnicity and risk for colorectal cancers showing somatic BRAF V600E mutation or CpG island methylator phenotype," Cancer Epidemiology Biomarkers and Prevention, vol. 17, no. 7, pp. 1774-1780, 2008.

[51] K. S. Boparai, J. B. Reitsma, V. Lemmens et al., "Increased colorectal cancer risk in first-degree relatives of patients with hyperplastic polyposis syndrome," Gut, vol. 59, no. 9, pp. 1222$1225,2010$.

[52] H. J. Bussey, "Gastrointestinal polyposis," Gut, vol. 11, no. 11, pp. 970-978, 1970.

[53] D. D. Buchanan, K. Sweet, M. Drini et al., "Phenotypic diversity in patients with multiple serrated polyps: a genetics clinic study," International Journal of Colorectal Disease, vol. 25, no. 6, pp. 703-712, 2010.

[54] E. Chow, L. Lipton, E. Lynch et al., "Hyperplastic polyposis syndrome: phenotypic presentations and the role of MBD4 and MYH," Gastroenterology, vol. 131, no. 1, pp. 30-39, 2006.

[55] D. C. Snover, D. J. Ahnen, R. W. Burt et al., "Serrated polyps of the colon and rectum and serrated polyposis," in WHO Classification of Tumours of the Digestive System, F. T. Bosman, 
F. Carneiro, R. H. Hruban, and etal, Eds., pp. 160-165, IARC Press, Lyon, France, 2010.

[56] J. R. Jass, H. Lino, A. Ruszkiewicz et al., "Neoplastic progression occurs through mutator pathways in hyperplastic polyposis of the colorectum," Gut, vol. 47, no. 1, pp. 43-49, 2000.

[57] J. R. Jass, J. Young, and B. A. Leggett, "Hyperplastic polyps and DNA microsatellite unstable cancers of the colorectum," Histopathology, vol. 37, no. 4, pp. 295-301, 2000.

[58] R. Burt and J. R. Jass, "Hyperplastic polyposis," in Pathology and Genetics of Tumours of the Digestive System, S. R. Hamilton and L. A. Aaltonen, Eds., pp. 135-136, IARC Press, Lyon, France, 2000.

[59] J. R. Jass, "Gastrointestinal polyposes: clinical, pathological and molecular features," Gastroenterology Clinics of North America, vol. 36, no. 4, pp. 927-946, 2007.

[60] M. Lockett and W. Atkin, "Hyperplastic polyposis (HPP): prevalence and cancer risk," Gastroenterology, vol. 120, no. 5, pp. A742-A742, 2001.

[61] K. S. Boparai, E. Dekker, S. van Eeden et al., "Hyperplastic polyps and sessile serrated adenomas as a phenotypic expression of MYH-associated polyposis," Gastroenterology, vol. 135, no. 6, pp. 2014-2018, 2008.

[62] R. K. Eun and K. Young-Ho, "Clinical application of genetics in management of colorectal cancer," Intestinal Research, vol. 12, no. 3, pp. 184-193, 2014.

[63] M. S. Sawhney, W. D. Farrar, S. Gudiseva et al., "Microsatellite instability in interval colon cancers," Gastroenterology, vol. 131, no. 6, pp. 1700-1705, 2006.

[64] R. Lazarus, O. E. Junttila, T. J. Karttunen, and M. J. Mäkinen, "The risk of metachronous neoplasia in patients with serrated adenoma," The American Journal of Clinical Pathology, vol. 123, no. 3, pp. 349-359, 2005.

[65] J. R. Jass, "Hyperplastic polyps of the colorectum-innocent or guilty?" Diseases of the Colon \& Rectum, vol. 44, no. 2, pp. 163166, 2001. 


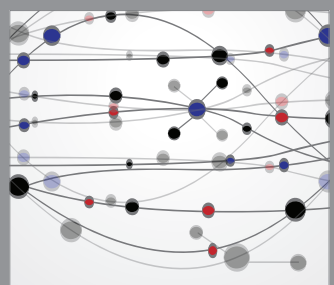

The Scientific World Journal
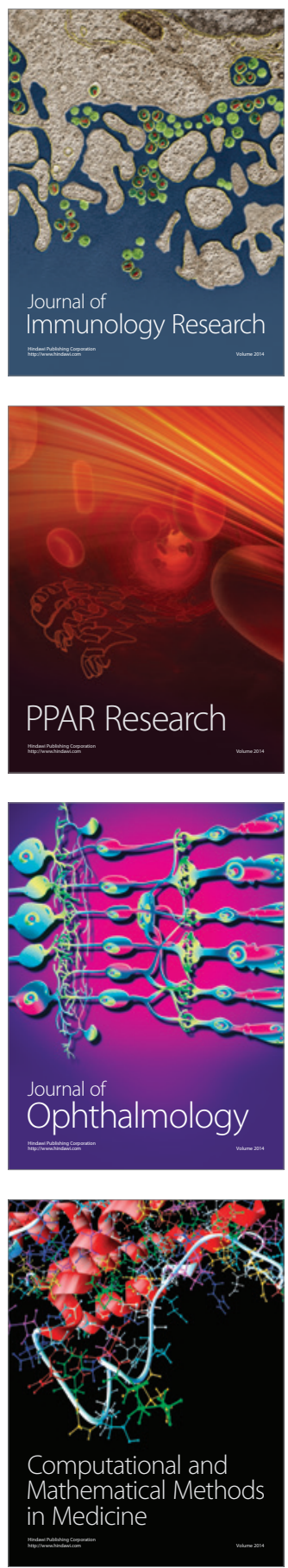

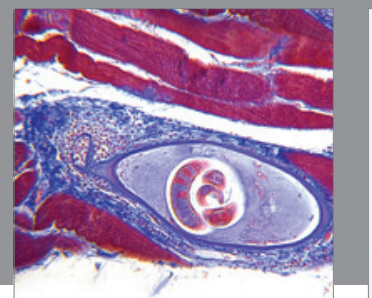

Gastroenterology

Research and Practice
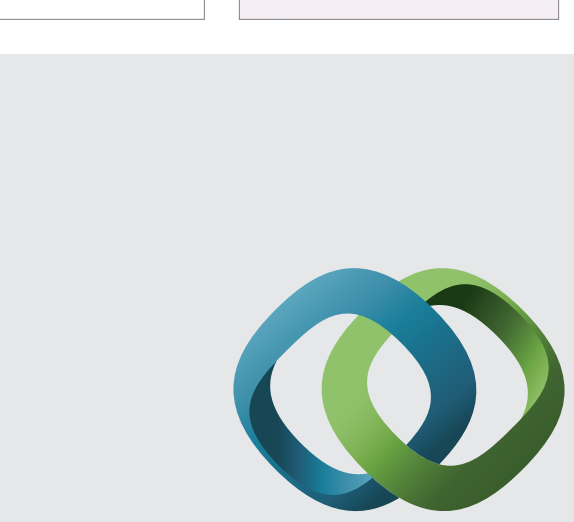

\section{Hindawi}

Submit your manuscripts at

http://www.hindawi.com
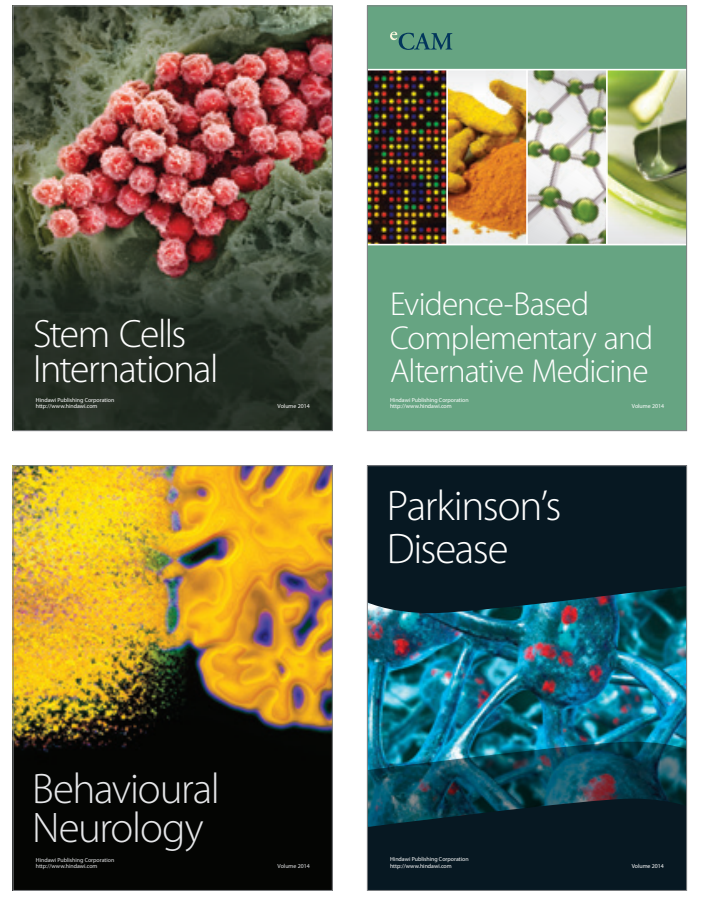
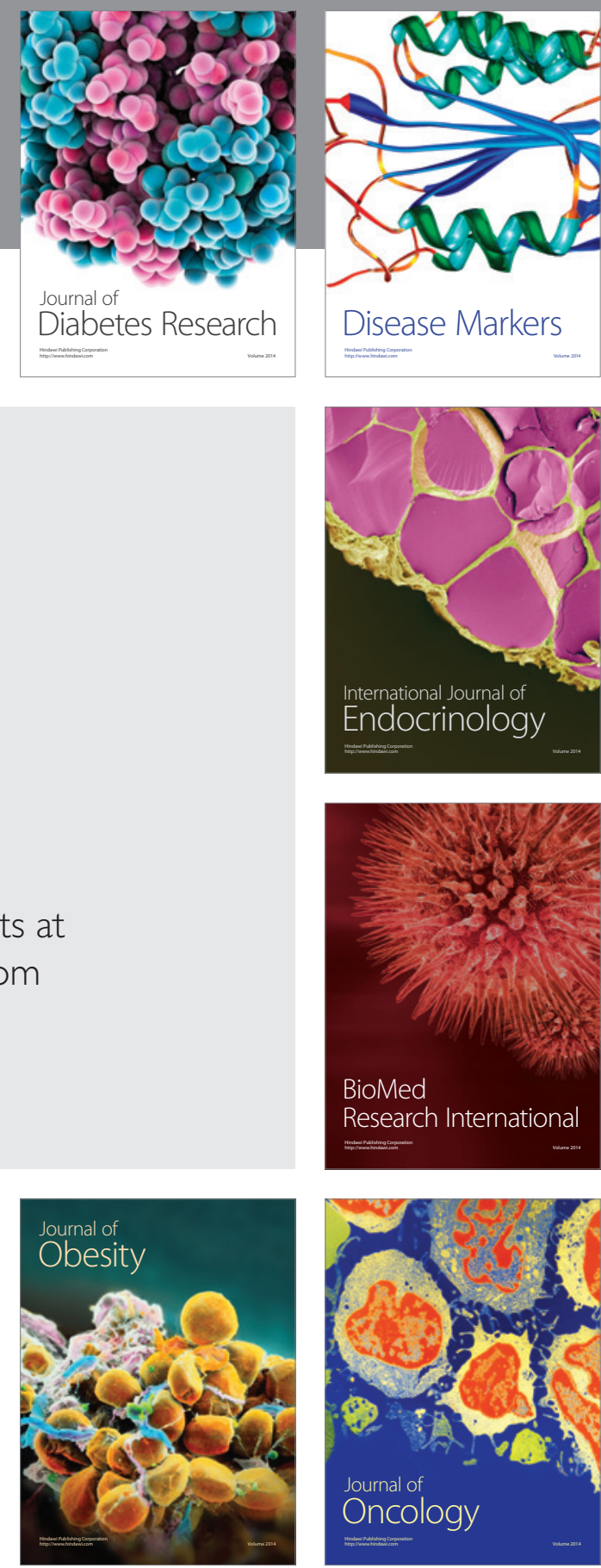

Disease Markers
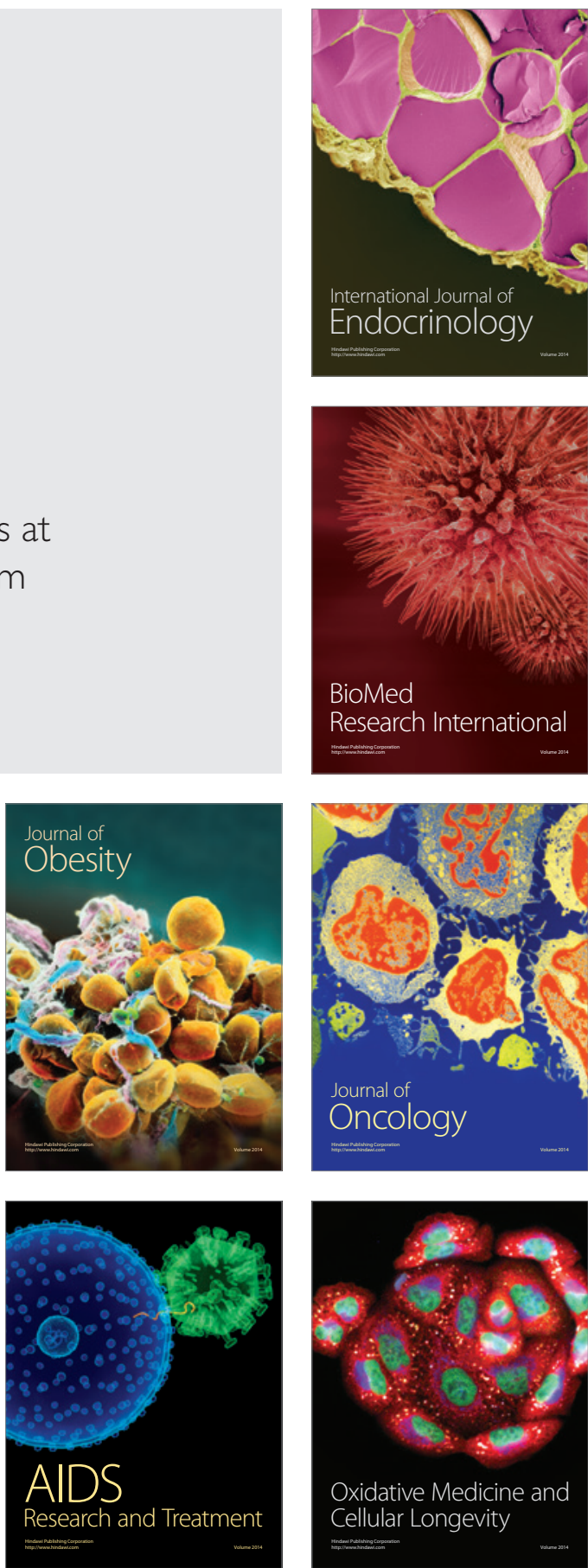